\title{
Whole-transcriptome causal network inference with genomic and transcriptomic data
}

\author{
Lingfei Wang and Tom Michoel
}

\begin{abstract}
Reconstruction of causal gene networks can distinguish regulators from targets and reduce false positives by integrating genetic variations. Its recent developments in speed and accuracy have enabled whole-transcriptome causal network inference on a personal computer. Here we demonstrate this technique with program Findr on 3,000 genes from the Geuvadis dataset. Subsequent analysis reveals major hub genes in the reconstructed network.
\end{abstract}

Key words: causal gene network, whole-transcriptome network, causal inference, genome-transcriptome variation

\section{Introduction}

Rapid developments in sequencing technologies have driven low the cost and high the throughput ([1]), with genomic and transcriptomic datasets from the same individuals increasingly publicly available (e.g. [2, 3]). The question now lies at the computational aspect, on how to fully exploit those datasets in order to address important biological and medical questions ([4]). Network-based approaches have received strong interests, especially from the clinical domain, where disease-related hub genes present attractive candidates for drug targeting ([5, 6] $)$.

In this chapter, we focus on the reconstruction of causal gene networks on genome and (whole-)transcriptome datasets. (See [7] for a review.) As opposed to co-expression networks, causal gene networks are directed, and can identify the reg-

Lingfei Wang

Division of Genetics and Genomics, The Roslin Institute, The University of Edinburgh, Midlothian EH25 9RG, Scotland, United Kingdom, e-mail: Lingfei.Wangeroslin.ed.ac.uk

Tom Michoel

Division of Genetics and Genomics, The Roslin Institute, The University of Edinburgh, Midlothian EH25 9RG, Scotland, United Kingdom 
ulator among two co-expressed genes, or the existence of a hidden confounder gene or a feedback loop. With more stringent statistical tests, causal inference can also reduce the notoriously high numbers of false positives in co-expression networks.

For this purpose, genomic variations, which are typically observed in cohort studies and recombinant inbred lines, can be integrated as causal anchors or instrumental variables. Similar to double-blinded randomized controlled trials, genomic variations define naturally randomized groupings of individuals that allow to infer the causal relations between quantitative traits, a principle also known as Mendelian randomization ([8]). To test for a causal relation from a candidate regulator to a target, Mendelian randomization seeks a shared upstream causal anchor that is associated to both. By assuming that the causal anchor can only affect the target through the regulator, the interaction would then be identified.

However, this assumption does not always hold for gene regulations. For example, even if genomic variations are limited to lie in the cis-regulatory region of the regulator (i.e. cis-expression quantitative trait loci; cis-eQTLs), they may still also be associated to other nearby genes, which in turn control the target. Consequently, existing studies and public softwares, namely Trigger ([9]) and CIT ([10]), proposed to test this assumption through a "conditional independence test". As was revealed in other studies, the conditional independence test cannot consider the existences of hidden confounders and technical variations $([11,7,12,13,14,15])$, which led to few discoveries of gene regulations. Additionally, neither software was efficient enough to handle the scale of modern datasets.

Recently in [14], we proposed alternative tests which are robust against confounders and technical variations. Implementational and statistical advances in the accompanying program Findr also resulted in almost 1,000,000 times of speed-up compared to CIT. This makes possible the reconstruction of whole-transcriptome causal gene networks, which can detect novel interactions by avoiding any preselection of genes.

In this chapter, we present a detailed protocol for the application of Findr, through an example where causal gene networks are inferred among 3000 genes from downsampled Geuvadis study data ([2]). This is supplemented with a brief outline of the methods implemented in Findr, and its future perspectives in method development and application domains.

\section{Notations and materials}

In this section, we briefly formalize the network inference question and establish the necessary computational environment for Findr. 


\subsection{Question formalization}

Consider genome-transcriptome variation data from $N$ unrelated individuals. After preprocessing and eQTL analysis, we have identified $G$ expressed genes (see Note 1), $g_{1}, g_{2}, \ldots, g_{G}$, in which the first $E \leq G$ genes (i.e. $g_{1}, g_{2}, \ldots, g_{E}$ ) have cis-eQTLs.

We denote the expression level (FPKM) of gene $g_{i}$ for individual $j$ as $g_{i, j}$, whose matrix form is

$$
\mathbf{G} \equiv\left(g_{i, j}\right), \quad i=1, \ldots, G, \quad j=1, \ldots, N .
$$

Similarly, for gene $g_{i}$, the genotype of its cis-eQTL for individual $j$ is defined as $e_{i, j}$, with the matrix form

$$
\mathbf{E} \equiv\left(e_{i, j}\right), \quad i=1, \ldots, E, \quad j=1, \ldots, N,
$$

where each genotype is limited by the number of alleles, $N_{a}$, as

$$
e_{i, j}=0,1, \ldots, N_{a}
$$

The unknown gene regulation network can be represented as the posterior probability of regulation between every pair of genes, given the observed data, as

$$
w_{i, j} \equiv P\left(g_{i} \rightarrow g_{j} \mid \mathbf{E}, \mathbf{G}\right), \quad i, j=1, \ldots, G, \quad i \neq j .
$$

Regulations are identified solely according to their expression and eQTL patterns, independent of the underlying mechanism or whether the regulation is direct (see Note 2).

Causal inference utilizes the cis-eQTL of every regulator gene to map the probability of regulation for all its possible targets. Therefore, genes without any ciseQTL $\left(g_{E+1}, \ldots, g_{G}\right)$ are regarded as only target genes but not as regulators, with

$$
w_{i, j}=0, \quad \text { for } i=E+1, \ldots, G .
$$

The expression levels of all possible regulators is also a sub-matrix of $\mathbf{G}$ as

$$
\mathbf{G}_{\mathrm{reg}} \equiv\left(g_{i, j}\right), \quad i=1, \ldots, E, \quad j=1, \ldots, N .
$$

Given the expression levels $\mathbf{G}$ and cis-eQTL genotypes $\mathbf{E}$, the question is to compute the probability of regulation matrix:

$$
\mathbf{W} \equiv\left(w_{i, j}\right), \quad i=1, \ldots, E, \quad j=1, \ldots, G .
$$




\subsection{The Findr program}

Findr (Fast Inference of Networks from Directed Regulations) is an efficient C library to reconstruct causal gene networks, whose methods can deal with the unique challenges in genomic and transcriptomic datasets, and are sketched in Sect. 4 . Findr provides interfaces in python2, R, and command line (see Note 3). Its efficient implementation and analytical calculation of null distributions (Sect.4.2.2p are pivotal to the speed-up of nearly 1 million times compared to existing programs [14]. This allows for whole-transcriptome causal network inference on modern datasets.

As a demonstrative example, we use the Findr $\mathrm{R}$ package with version 1.0.3 ([16]) in this chapter (see Note 4).

\subsection{Computing environment}

At the time of writing, the latest Findr R package (version 1.0.3) requires the following computing environment:

- A modern personal computer, or a high performance computing environment (see Note 5).

- A modern Linux or Mac operating system.

- The GCC compiler (see Note 6).

- A command-line environment (to install Findr).

- A recent $\mathrm{R}$ language environment ( $\mathrm{R}, \mathrm{RStudio}$, etc).

\section{Whole-transcriptome causal network from the Geuvadis dataset}

The Geuvadis project [2] measured genome-wide genotypes and gene expression levels in 465 human lymphoblastoid cell line samples. Using this dataset as an example, here we reconstruct a causal gene network with Findr in R.

\subsection{Install Findr}

The latest version of Findr (see Note 7) can be downloaded and installed with the following lines in command-line environment (see Note 8):

\#Comments above a command explains its function 
\#Comments below a command (if present) shows its expected output

\#Download Findr R package from github (see Note 9)

git clone https://github.com/lingfeiwang/findr-R.git

\#Install Findr

cd findr-R \&\& R CMD INSTALL findr

\subsection{Prepare data}

Here we reconstruct a causal gene regulation network among 3000 genes in which 1000 have cis-eQTLs. The dataset was downsampled from the Geuvadis project (see Note 10). In R, the Findr library and the downsampled Geuvadis dataset can be loaded with:

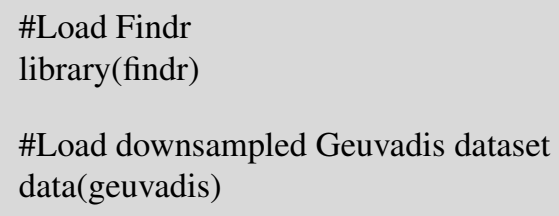

\subsection{Reconstruct network}

Network inference is performed with the function findr.pij_gassist, taking $\mathbf{E}, \mathbf{G}_{\text {reg }}$, and $\mathbf{G}$ as input and returning $\mathbf{W}$ as ouput (see Note 11):

\#Reconstruct causal gene network

$\mathrm{w}=$ findr.pij_gassist (geuvadis\$dgt,geuvadis $\$ d t$,geuvadis $\$ \mathrm{dt} 2$, nodiag=TRUE)

\#Examine output dimension

$\operatorname{print}(\operatorname{dim}(w))$

\#[1] 10003000

The computation takes about one second on a modern desktop computer, and scales linearly with the numbers of regulators, targets, and individuals. 


\subsection{Analyze and visualize network}

To demonstrate the properties of the reconstructed causal network for human lymphoblastoid cell lines, we briefly analyze and visualize it below:

\subsubsection{Regulation probabilities}

The distribution of posterior regulation probability $P\left(g_{i} \rightarrow g_{j} \mid \mathbf{E}, \mathbf{G}\right)$ is visualized in Fig. 1 with code (see Note 12):

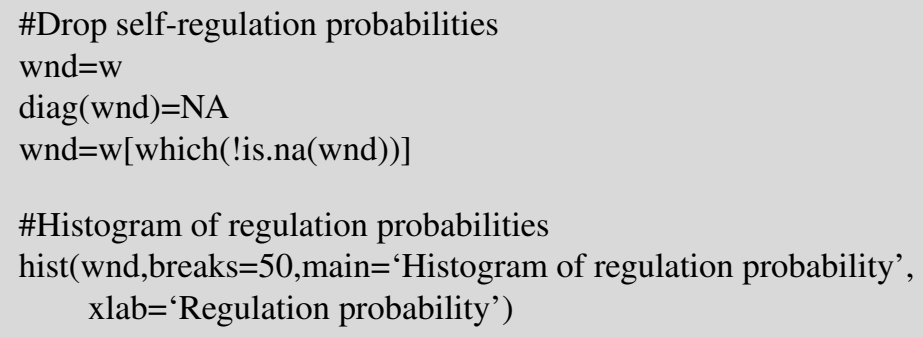

\subsubsection{Out-degree distribution}

The distribution of out-degree is visualized in Fig. 2 with code:

\#Threshold network to $90 \%$ confidence level

threshold $=0.9$

net $=\mathrm{w}>=$ threshold

\#Histogram of out-degree

odeg=rowSums(net)

vals=table(odeg)

plot(names(vals),vals, log='y',main='Distribution of

out-degree', xlab='Out-degree', $y l a b=$ 'Number of regulator genes')

\subsubsection{Top hub gene list}

The list of top hub genes (by out-degree) is obtained below: 
\#Find top 5 hub genes, and their numbers of targets

ntop $=5$

odego $=$ odeg[order(odeg,decreasing=TRUE)]

print(odego[1:ntop])

\# ZNF77 SP2

\# $155 \quad 146$

PEA15

ZNF580

C6orf106

28

7

6

\subsubsection{Network visualization in Cytoscape}

The reconstructed network can be exported to a csv file for visualization in Cytoscape (see Note 13), with the following code:

\#Convert causal network to sparse format

dat=NULL

for (i in $1: \operatorname{dim}($ net) $[1])$

if $(\operatorname{any}($ net $[i])$, dat $=\operatorname{cbind}($ dat,rbind(rownames(net) $[i]$, names $($ which $($ net $[i])))$,

dat $=\mathrm{t}($ dat $)$

colnames(dat)=c('source', 'target')

$\operatorname{print}(\operatorname{dat}[1: 2]$,

\# source target

\#[1,] "ARHGEF35" "OR2A1"

\#[2,] "ARHGEF35" "OR2A42"

\#Export sparse network to dat.csv

write.csv(dat, 'dat.csv',row.names=FALSE,quote=FALSE)

Cytoscape can then import the network in dat.csv and visualize it. The largest connected component is shown in Fig. 3

\section{Statistical Methods}

In this section, we outline the statistical methods used in Findr. (For details, see [14].) 
Lingfei Wang and Tom Michoel

\subsection{Data normalization}

To satisfy the assumptions of linear dependency and normal noise distribution, and also to remove outliers (see Note 14), the expression levels of each gene are transformed to follow the standard normal distribution, based on the expression level ranking across individuals. Each gene is normalized separately.

\subsection{Causal inference subtests}

Consider all possible regulatory relations between the triplet $(A, B, C)$, in which $B$ is the regulator gene, $A$ is its cis-eQTL, and $C$ is a potential target gene. Causal inference performs three subtests in Table 1 (see Note 15) to narrow down their relation to contain $A \rightarrow B \rightarrow C$, but with a false positive rate as low as possible. Each subtest compares a null and an alternative hypothesis, by first performing a likelihood ratio test, then converting the likelihood ratio into p-values, and finally computing the posterior probability of alternative hypotheses given the observed data, which is equivalent with the local False Discovery Rate (FDR). This is similar with Genome Wide Association Studies, in which the Pearson correlation (equivalent with likelihood ratio) is first computed, and then converted into p-values and FDRs.

\subsubsection{Likelihood ratio tests}

In Table 1, each graph represents a probablistic dependency model among the $(A, B, C)$ triplet. The expression level of each gene is modelled as following a normal distribution, whose mean depends additively on all its parents. The dependency is linear on other gene expressions, and categorical on genotypes. Based on the normally distributed models, the likelihood ratio between the alternative and the null hypotheses can be computed for each subtest.

\subsubsection{P-values}

For each subtest, the null distribution of likelihood ratio may be obtained either by simulation or analytically. Regardless of the method, likelihood ratios can then be converted into p-values according to their rankings in the null distribution. In [14], we found that the null distribution can be computed analytically, therefore avoiding simulations and accelerating the computations in Findr by $\sim 1000$ times. 


\subsubsection{Posterior probabilities of alternative hypotheses}

P-values can be further reformulated into the posterior probabilities of alternative hypotheses, according to [17, 9]. As opposed to FDRs, the combination of subtests requires posterior probabilities which correspond to local FDRs (see Note 16). Findr implements a simplified estimator (see Note 17), with the resulting posterior probabilities denoted as

$$
p_{i, j}^{(k)} \equiv P\left(\text { alternative hypothesis in subtest } k \text { for } A=e_{i}, B=g_{i}, C=g_{j} \mid \mathbf{E}, \mathbf{G}\right) .
$$

\subsection{Subtest combination}

Findr computes the final probability of regulation by combining the subtests as:

$$
w_{i, j} \equiv \frac{1}{2}\left(p_{i, j}^{(1)} p_{i, j}^{(2)}+p_{i, j}^{(3)}\right) .
$$

By combining the secondary linkage and controlled tests, the first term verifies that the correlation between $g_{i}$ and $g_{j}$ is not entirely due to pleiotropy. By replacing the conditional independence test in [9] with the controlled test, this combination is robust against hidden confounders and technical variations.

On the other hand, the relevance test in the second term can identify interactions that arise from the indirect effect $e_{i} \rightarrow g_{i} \rightarrow g_{j}$ but are too weak to be detected by the secondary linkage test. However, in such cases the direction of regulation cannot be determined. The coefficient $\frac{1}{2}$ simply assigns half of the probability to each direction.

\section{Notes}

1. The whole chapter is equally applicable on gene isoforms.

2. However, direct regulations tend to have stronger significance than the indirect regulation they form, if the relevant genes have similar levels of technical variations.

3. URLs for Findr library and interfaces:

- Library: https://github.com/lingfeiwang/findr

- Command line interface:https://github.com/lingfeiwang/findr-bin

- Python2 interface:/https://github.com/lingfeiwang/findr-python

- R package:https://github.com/lingfeiwang/findr-R

4. Findr's python 2 and command-line interfaces have the same functionality with similar computing environment requirements. 
5. The computer memory (RAM) limits the size of data Findr can process. Findr's python 2 and command-line interfaces can automatically split the dataset according to the specified memory usage limit. The $\mathrm{R}$ package requires manual division. A modern computer with 4GB of RAM can handle whole-transcriptome network inference for hundreds of individuals, with thousands of genes having cis-eQTLs and tens of thousands having not.

6. GCC is already installed on most Linux machines. On Mac OS, Apple's compiler may pretend to be GCC, so Findr may fail to install. For the solution, see FAQ in https://github.com/lingfeiwang/findr/blob/master/doc.pdf. GCC can be downloaded from https: / / gcc.gnu.org.

7. To exactly reproduce the example, Findr 1.0.3 can be downloaded from [16].

8. Detailed installation instructions and FAQs are available in the full manual at https://github.com/lingfeiwang/findr/blob/master/doc.pdf, or the doc.pdf file of the corresponding version.

9. Without git, the latest version of Findr can also be downloaded from https:// github.com/lingfeiwang/findr-R/archive/master.zip. The user then needs to uncompress it and enter the corresponding folder before installation.

10. The original, full Geuvadis dataset can be downloaded from ArrayExpress (https : //www.ebi.ac.uk/arrayexpress/), in accessions E-GEUV-1 and EGEUV-2. In this analysis, 360 unique European individuals of 465 in total are considered. Geuvadis reported 23722 genes expressed (in $\geq 50 \%$ individuals) after QC. Genetic loci without any variation were discarded. After that, the Geuvadis QTL analysis identified 3172 genes with at least one significant cis-eQTL.

Findr includes a random subset of these data for illustration purposes, totalling 1000 genes with cis-eQTLs and 2000 more without cis-eQTLs. For each of the 1000 genes, the genotypes of its strongest cis-eQTL are also included.

The same network analysis can be performed on other datasets, such as on the full Geuvadis dataset to reconstruct the whole-transcriptome causal gene networks among 23722 genes. This requires an already performed eQTL analysis for the dataset, either from softwares such as matrixeQTL ([18]) or fastQTL ([19]), or from existing studies.

11. Function description for findr.pij_gassist(dg,dt,dt2,na=NULL,nodiag=FALSE):

- dg: Input integer matrix $\mathbf{E}$ of cis-eQTL genotype data, as defined in Eq 2 . The element $[i, j]$ is the genotype value $\left(0,1, \ldots, N_{a}\right)$ of the cis-eQTL of regulator gene $i$ for individual $j$.

- dt: Input double matrix $\mathbf{G}_{\text {reg }}$ of regulator gene expression level data, as defined in Eq[6. The element $[i, j]$ is the expression level of regulator $i$ for individual $j$.

- dt2: Input double matrix $\mathbf{G}$ of all gene expression level data, as defined in Eq 1. The element $[i, j]$ is the expression level of gene $i$ for individual $j$.

- na: The number of alleles $N_{a}$. If unspecified, it is automatically detected as the maximum value of $\mathrm{dg}$. 
- nodiag: This function can infer networks for regulators that either should or should not be regarded as targets. In the earlier case, the regulators should also appear before other genes as targets, and nodiag should be TRUE. In the latter case, there should be no overlap between regulators and targets, with nodiag=FALSE.

- Return value: Output double matrix $\mathbf{W}$ of inferred probability of regulation, as defined in $\mathrm{Eq} 7$ The element $[i, j]$ is the probability of regulation from regulator $i$ to target $j$ after observing the input data.

12. The peak at regulation probability around 0.5 is due to correlated regulator and target genes, whose regulation direction cannot be determined with the cis-eQTL of the regulator. In such cases, the novel combination of causal inference tests assumes a half probability for each direction.

13. http://www.cytoscape.org/

14. Although the data normalization step attempts to transform gene expression levels into the standard normal distribution, ill-distributed datasets may still cause underperformance from the method and should therefore be analyzed with extra care. Examples may include a large proportion of ties in gene expression levels, from single-cell transcriptomics or sparsely expressed genes.

15. Here we omit the primary linkage test, comparing $A \rightarrow B$ against $A \quad B$, because the cis-eQTL $A$ is assumed to be significant. These tests are also numbered differently with [14].

16. For the difference between FDR and local FDR, see [20].

17. Findr also skips the computation of P-values when deriving the posterior probabilities using the null distribution. For more stringent local FDR conversion with Grenander estimator, the user can first compute p-values within Findr (findr.pijs_gassist_pv) and then use other softwares (e.g. fdrtool in R from [20]) to obtain local FDRs.

\section{Future perspectives}

The reconstruction of causal networks can be potentially extended to various data types. For example, other causal anchors to infer causal gene networks may include epigenetic markers, copy number variations, and perturbation screens. By targeting tissue-specific or species-specific genes, Findr may also reconstruct cross-tissue or host-pathogen/microbiota causal gene networks. The same analysis may also apply on gene isoforms, proteome, etc, to reconstruct multi-omics causal networks. By considering different distributions of technical variation, the very same causal inference may also infer cell type-specific causal networks from single-cell datasets. Each of those perspectives contains its unique challenges that are worth addressing in the future. However, the statistical and computational frameworks have already been laid down. 
Acknowledgements Development of Findr was supported by grants from the BBSRC [BB/J004235/1, BB/M020053/1].

\section{References}

1. Sara Goodwin, John D. McPherson, and W. Richard McCombie. Coming of age: ten years of next-generation sequencing technologies. Nature Reviews Genetics, 17(6):nrg.2016.49, May 2016. URL: https://www.nature.com/articles/nrg.2016.49 doi: $10.1038 / \mathrm{nrg} .2016 .49$

2. Tuuli Lappalainen, Michael Sammeth, Marc R. Friedlnder, Peter A. C. t Hoen, Jean Monlong, Manuel A. Rivas, Mar Gonzlez-Porta, Natalja Kurbatova, Thasso Griebel, Pedro G. Ferreira, Matthias Barann, Thomas Wieland, Liliana Greger, Maarten van Iterson, Jonas Almlf, Paolo Ribeca, Irina Pulyakhina, Daniela Esser, Thomas Giger, Andrew Tikhonov, Marc Sultan, Gabrielle Bertier, Daniel G. MacArthur, Monkol Lek, Esther Lizano, Henk P. J. Buermans, Ismael Padioleau, Thomas Schwarzmayr, Olof Karlberg, Halit Ongen, Helena Kilpinen, Sergi Beltran, Marta Gut, Katja Kahlem, Vyacheslav Amstislavskiy, Oliver Stegle, Matti Pirinen, Stephen B. Montgomery, Peter Donnelly, Mark I. McCarthy, Paul Flicek, Tim M. Strom, The Geuvadis Consortium, Hans Lehrach, Stefan Schreiber, Ralf Sudbrak, ngel Carracedo, Stylianos E. Antonarakis, Robert Hsler, Ann-Christine Syvnen, Gert-Jan van Ommen, Alvis Brazma, Thomas Meitinger, Philip Rosenstiel, Roderic Guig, Ivo G. Gut, Xavier Estivill, and Emmanouil T. Dermitzakis. Transcriptome and genome sequencing uncovers functional variation in humans. Nature, 501(7468):506-511, September 2013. URL: https://www.nature.com/nature/journal/v501/ n7468/full/nature12531.html ?error=cookies_not_supported\&code= 20722c34-2b4b-45b1-b47d-17350071fccb doi:10.1038/nature12531

3. The GTEx Consortium. The Genotype-Tissue Expression (GTEx) pilot analysis: Multitissue gene regulation in humans. Science, 348(6235):648-660, May 2015. URL: http://science.sciencemag.org/content/348/6235/648, doi:10. $1126 /$ science.1262110

4. Euan A. Ashley. Towards precision medicine. Nature Reviews Genetics, 17(9):507522, September 2016. URL: https://www.nature.com/nrg/journal/v17/ n9/full/nrg.2016.86.html ?error=cookies_not_supported\&code $=$ 5128c118-9ba3-41d2-b095-2294b095889f. doi:10.1038/nrg.2016.86

5. Eric E. Schadt, Stephen H. Friend, and David A. Shaywitz. A network view of disease and compound screening. Nature Reviews Drug Discovery, 8(4):286295, April 2009. URL: https://www.nature.com/nrd/journal/v8/ n4/full/nrd2826.html?error=cookies_not_supported\&code= bfa87d2b-c137-4078-b1bb-bc71df321f89\#top doi:10.1038/nrd2826

6. HusainA. Talukdar, Hassan ForoughiAsl, RajeevK. Jain, Raili Ermel, Arno Ruusalepp, Oscar Franzn, BrianA. Kidd, Ben Readhead, Chiara Giannarelli, JasonC. Kovacic, Torbjrn Ivert, JoelT. Dudley, Mete Civelek, AldonsJ. Lusis, EricE. Schadt, Josefin Skogsberg, Tom Michoel, and JohanL. M. Bjrkegren. Cross-Tissue Regulatory Gene Networks in Coronary Artery Disease. Cell Systems, 2(3):196-208, March 2016. URL:/http://www.sciencedirect.com/science/article/pii/ S2405471216300321. doi:10.1016/j.cels.2016.02.002

7. Matthew V. Rockman. Reverse engineering the genotypephenotype map with natural genetic variation. Nature, 456(7223):738-744, December 2008. URL: https://www.nature.com/nature/journal/v456/n7223/ abs/nature07633.html?error=cookies_not_supported\&code= b25f94c5-510f-48a9-872d-fcb1cc04b8e2. doi:10.1038/nature07633

8. Debbie A. Lawlor, Roger M. Harbord, Jonathan A. C. Sterne, Nic Timpson, and George Davey Smith. Mendelian randomization: Using genes as instruments for mak- 
ing causal inferences in epidemiology. Statistics in Medicine, 27(8):1133-1163, April 2008. URL: http://onlinelibrary.wiley.com/doi/10.1002/sim.3034/ abstract. doi:10.1002/sim.3034

9. Lin S. Chen, Frank Emmert-Streib, and John D. Storey. Harnessing naturally randomized transcription to infer regulatory relationships among genes. Genome Biology, 8:R219, 2007. URL: http://dx.doi.org/10.1186/gb-2007-8-10-r219, doi:10. $1186 / \mathrm{gb}-2007-8-10-r 219$

10. Joshua Millstein, Gary K. Chen, and Carrie V. Breton. cit: hypothesis testing software for mediation analysis in genomic applications. Bioinformatics, 32(15):2364-2365, August 2016. URL: https://academic.oup.com/bioinformatics/article-lookup/ doi/10.1093/bioinformatics/btw135, doi:10.1093/bioinformatics/ btw135

11. Sander Greenland. THE EFFECT OF MISCLASSIFICATION IN THE PRESENCE OF COVARIATES. American Journal of Epidemiology, 112(4):564-569, October 1980. URL: https://academic.oup.com/aje/article/112/4/564/59323. doi: 10.1093/oxfordjournals.aje.a113025

12. Yang Li, Bruno M. Tesson, Gary A. Churchill, and Ritsert C. Jansen. Critical reasoning on causal inference in genome-wide linkage and association studies. Trends in Genetics, 26(12):493-498, December 2010. URL: http: //wWw.sciencedirect.com/science/article/pii/s0168952510001885. doi:10.1016/j.tig.2010.09.002

13. Stephen R. Cole, Robert W. Platt, Enrique F. Schisterman, Haitao Chu, Daniel Westreich, David Richardson, and Charles Poole. Illustrating bias due to conditioning on a collider. International Journal of Epidemiology, 39(2):417-420, April 2010. URL: https://academic.oup.com/ije/article/39/2/417/680407, doi: $10.1093 /$ i je/dyp334

14. Lingfei Wang and Tom Michoel. Efficient and accurate causal inference with hidden confounders from genome-transcriptome variation data. PLOS Computational Biology, 13(8):e1005703, August 2017. URL: http://journals.plos. org/ploscompbiol/article?id=10.1371/journal.pcbi.1005703. doi:10.1371/journal.pcbi.1005703

15. Gibran Hemani, Kate Tilling, and George Davey Smith. Orienting The Causal Relationship Between Imprecisely Measured Traits Using Genetic Instruments. bioRxiv, page 117101, March 2017. URL: https://www.biorxiv.org/content/early/2017/03/15/ 117101. doi:10.1101/117101

16. Lingfei Wang. Findr-R 1.0.3, October 2017. URL: https://doi.org/10.5281/ zenodo.1036757. doi:10.5281/zenodo.1036757

17. John D. Storey and Robert Tibshirani. Statistical significance for genomewide studies. Proceedings of the National Academy of Sciences, 100(16):9440-9445, August 2003. URL: http://www.pnas.org/content/100/16/9440 doi:10.1073/pnas. 1530509100

18. Andrey A. Shabalin. Matrix eQTL: ultra fast eQTL analysis via large matrix operations. Bioinformatics, 28(10):1353-1358, May 2012. URL: https:// academic.oup.com/bioinformatics/article/28/10/1353/213326 doi: 10.1093/bioinformatics/bts163

19. Halit Ongen, Alfonso Buil, Andrew Anand Brown, Emmanouil T. Dermitzakis, and Olivier Delaneau. Fast and efficient QTL mapper for thousands of molecular phenotypes. Bioinformatics, 32(10):1479-1485, May 2016. URL: https://academic. oup.com/bioinformatics/article/32/10/1479/1742545 doi:10.1093/ bioinformatics/btv722

20. Korbinian Strimmer. fdrtool: a versatile $\mathrm{R}$ package for estimating local and tail area-based false discovery rates. Bioinformatics, 24(12):1461-1462, June 2008. URL: https:// academic.oup.com/bioinformatics/article/24/12/1461/196272 doi: $10.1093 /$ bioinformatics/btn209 
Table 1 Three subtests of causal inference performed to test the gene regulation $B \rightarrow C$, with the cis-eQTL of $B$ as the causal anchor $A$. These tests are numbered, named, and defined in terms of null and alternative hypothese as shown. Arrows in a hypothesis indicate directed regulatory relations. (From [14].)

\begin{tabular}{cc|ccc} 
Test ID & Test name & $\begin{array}{c}\text { Null } \\
\text { hypothesis }\end{array}$ & $\begin{array}{c}\text { Alternative } \\
\text { hypothesis }\end{array}$ \\
\hline 1 & $\begin{array}{c}\text { Secondary } \\
\text { (linkage) }\end{array}$ & A & C & Controlled \\
3 & Relevance &
\end{tabular}




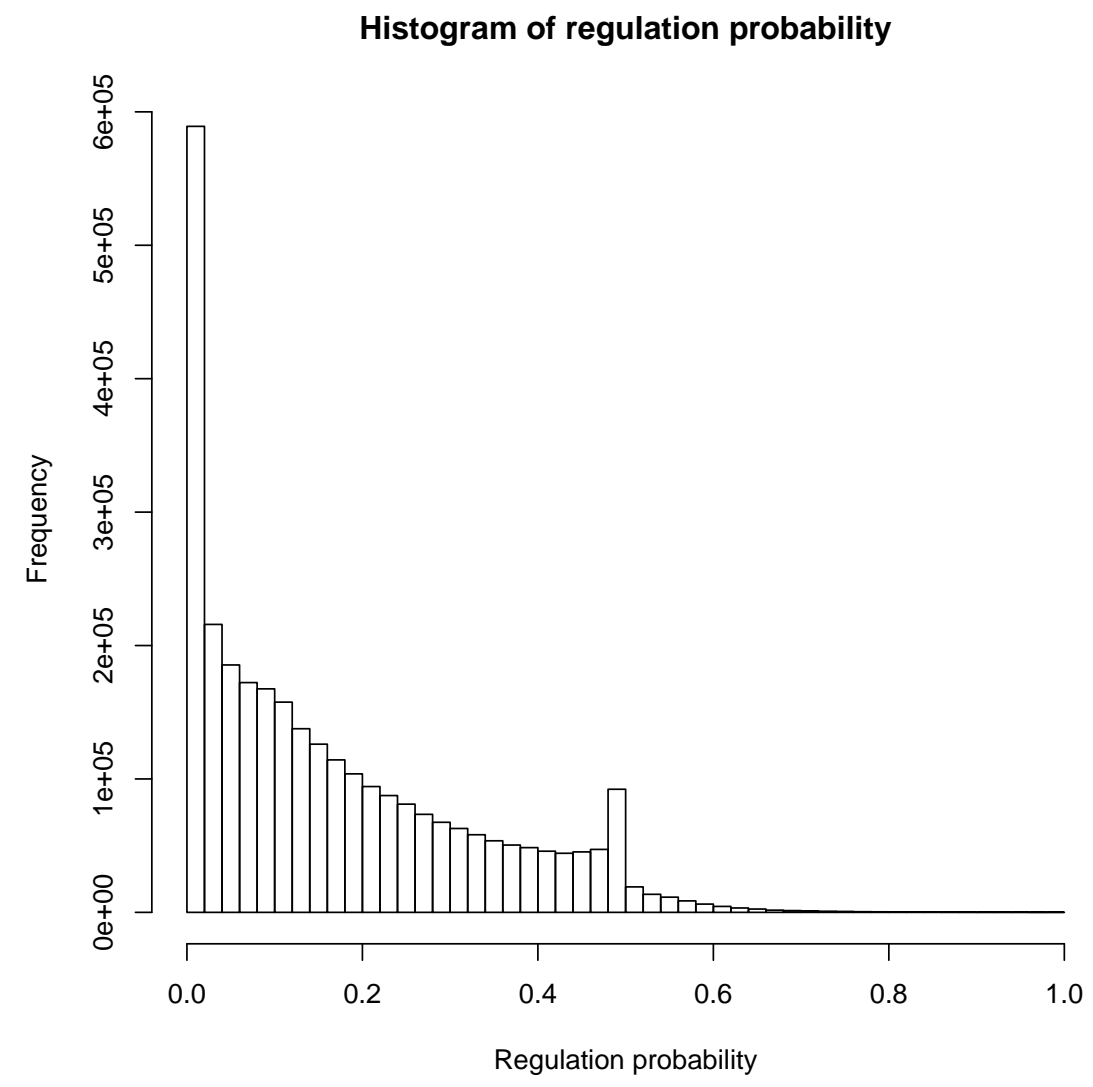

Fig. 1 The histogram of regulation probability (off-diagonal elements of $\mathbf{W}$ ) of the causal gene network reconstructed from the downsampled Geuvadis dataset. 


\section{Distribution of out-degree}

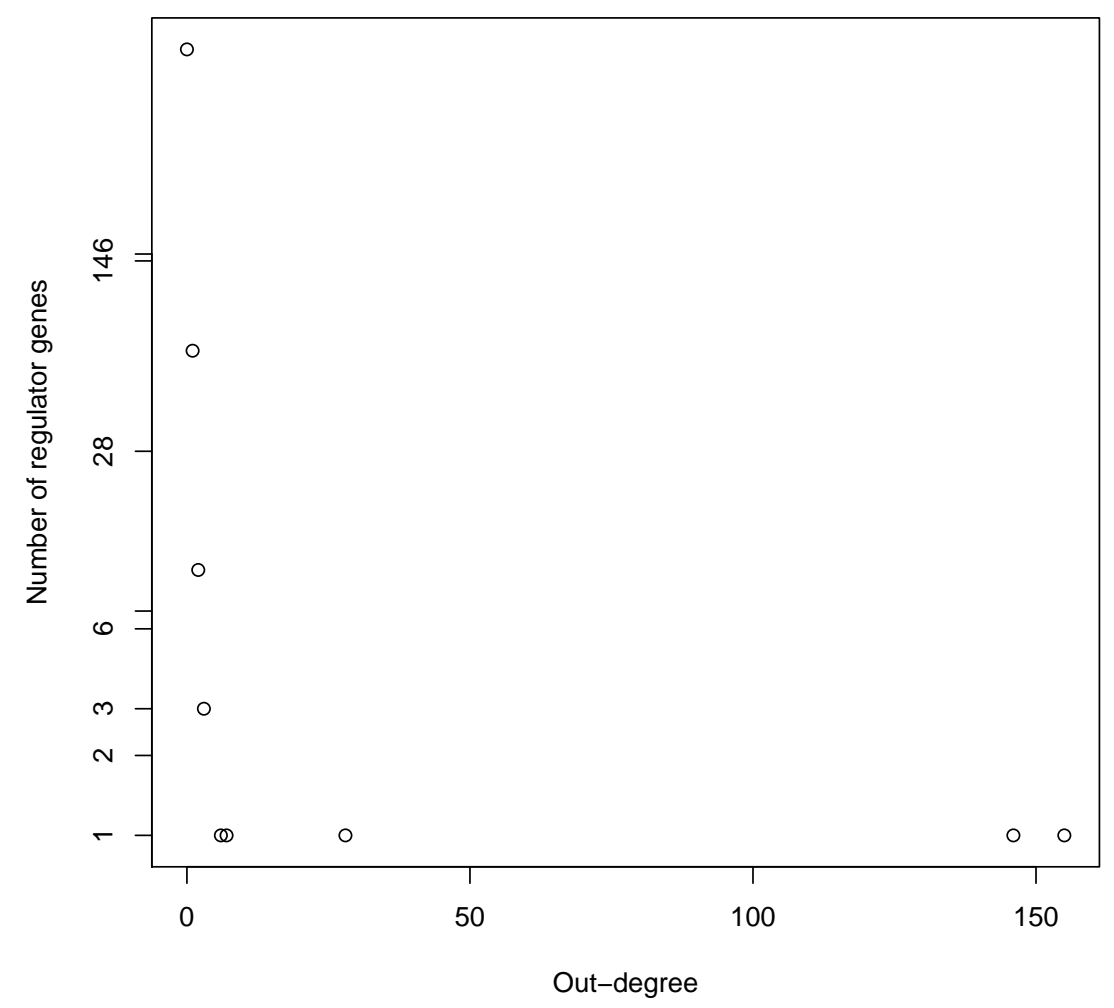

Fig. 2 The histogram of out-degree of regulator genes in the thresholded causal gene network reconstructed from the downsampled Geuvadis dataset. 


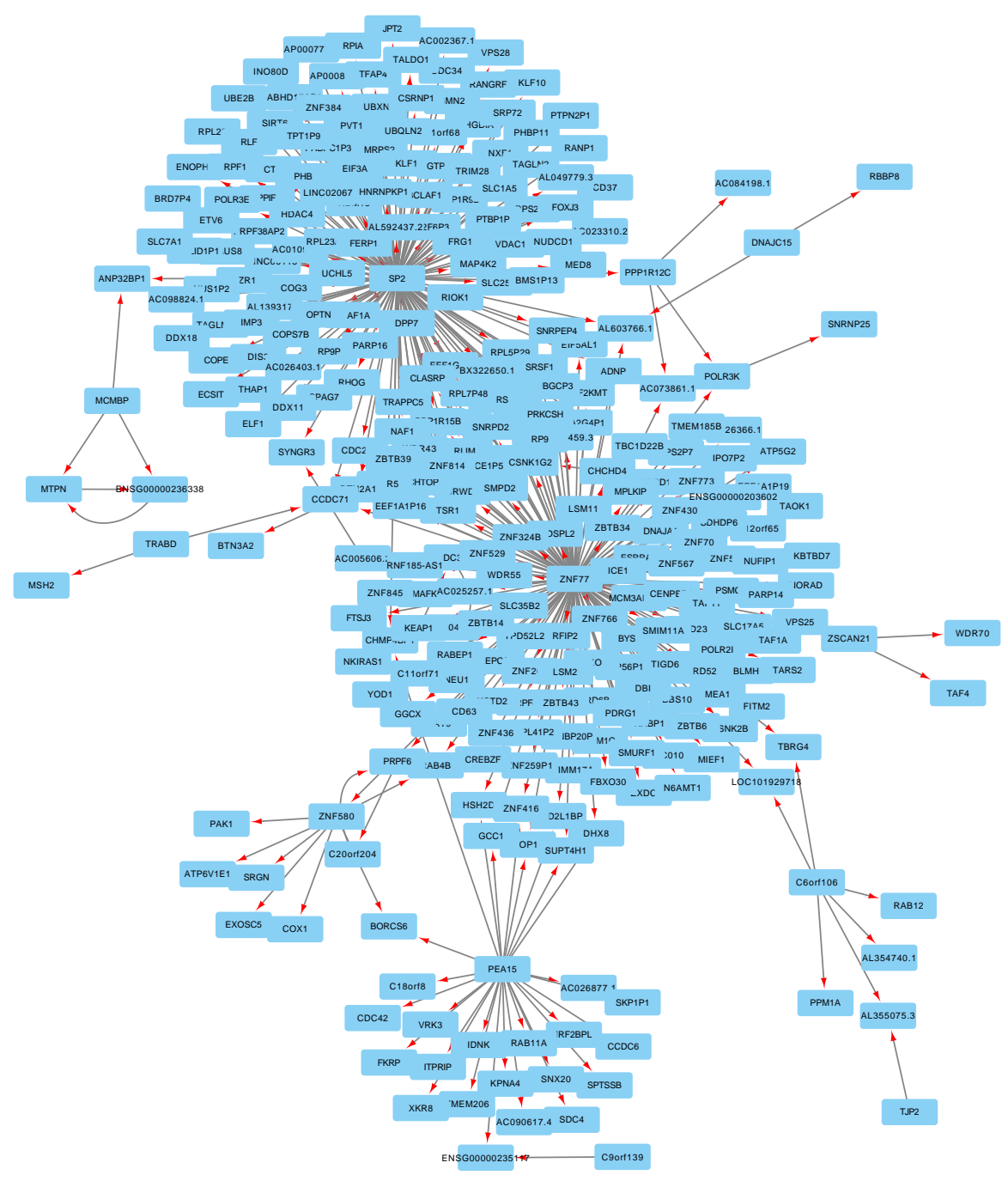

Fig. 3 The largest connected component of the reconstructed and thresholded regulation network from the downsampled Geuvadis dataset, as visualized in Cytoscape. Of the 3000 genes, 308 are in this connected component with 366 regulations. 